\title{
The EUVE Observations of Dwarf Novae
}

\author{
KNOX S. LONG
}

Space Telescope Science Institute, 3700 San Martin Drive, Baltimore, MD 21218, USA

In the standard theory of dwarf novae in outburst, the boundary layer region between the inner edge of the accretion disk and the white dwarf surface radiates primarily in the extreme ultraviolet. Using $E U V E$, observers have been able to obtain spectra with sufficient spectral resolution to characterize accurately the emission from several dwarf novae in outburst, including U Geminorum and SS Cygni. I present an overview of the observations and early analyses of the dwarf nova observations. The spectra obtained of dwarf novae are complex compared to the EUV spectra of magnetic cataclysmic variables and single white dwarfs. Detailed spectral modeling of an expanding atmosphere will most like be required to fully understand the spectra. Nevertheless, we already know there were significant differences in the effective temperatures and other properties of the EUV emissions. If we assume the EUV emission arises primarily from the boundary layer and parameterize the EUV spectrum in terms of a blackbody, then for $\mathrm{U}$ Gem the derived boundary luminosity is comparable to the disk luminosity, consistent with the standard theory, and the minimum size of the emitting region is about that of the white dwarf surface. The count rates from $U$ Gem were modulated strongly with the orbital period; differences in the shape of the spectrum in eclipse and out of eclipse suggest that while the bulk of the emission arose from the vicinity of the white dwarf, there was an extended source of emission as well. For SS Cyg, however, the derived boundary layer luminosity was a small fraction of the disk luminosity. In $U$ Gem, the effective temperature dropped during the decline from outburst. In contrast, in SS Cyg, the effective temperature remained constant as the count rate rose by a factor of 100 and the effective size increased. Thus while the observations of $U$ Gem seem broadly consistent with the standard theory for the boundary layer emission from dwarf novae, SS Cyg appears to present fundamental challenges to that theory.

\section{Introduction}

Dwarf novae are mass-exchanging binary star systems containing a white dwarf (WD) and a relatively normal companion star. In dwarf novae, the magnetic field is low and accretion occurs by means of a viscous disk. Dwarf novae are characterized by quasiperiodic outbursts of 3-5 magnitudes at visible wavelengths. Although alternatives have been proposed, the outbursts are probably due to a thermal instability associated with a dramatic change in the opacity of disk material near $10,000 \mathrm{~K}$, in essence, the temperature for hydrogen and helium ionization (Osaki 1974; Meyer \& Meyer-Hofmeister 1982). In outburst in the "standard" theory, the disk is optically thick and the effective temperature of the disk is typically $20,000 \mathrm{~K}-40,000 \mathrm{~K}$, depending on the accretion rate. In outburst, most of the luminosity of the disk emerges in the UV. In contrast, in quiescence, the disk should be optically thin and cool $(2,000 \mathrm{~K}$ to $7,000 \mathrm{~K})$ and very little UV emission should emerge from the disk (Cannizzo \& Wheeler 1984).

In a steady-state accretion disk, half the energy of accretion (GMM/r) is radiated as matter traverses the disk, but the other half reaches the inner edge of the accretion disk in the form of kinetic energy and is radiated away in a transition region which is known as the boundary layer. Because the boundary layer region is smaller than the accretion disk, the effective temperature of the boundary layer is higher. In outburst the boundary layer region should be optically thick, have an effective temperature of $100,000-300,000$ $\mathrm{K}$, and radiate primarily in the EUV (Pringle 1977). In contrast, in quiescence, primarily due to the density dependence of the volume emissivity, the boundary layer should be be optically thin and relatively hot $\left(10^{7} \mathrm{~K}\right)$. 

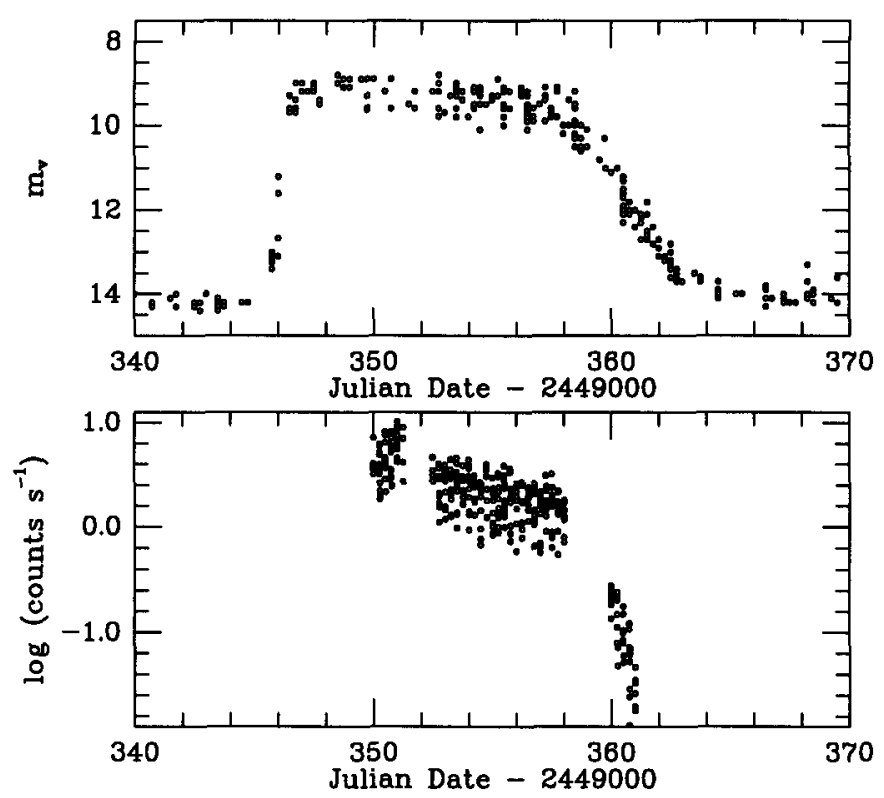

FIgURE 1. The AAVSO lightcurve (upper panel) and the count rate observed in the SW spectrometer (lower panel) for U Gem. The short term variations in the EUVE lightcurve are largely due to changes in the flux as a function of orbital phase.

$E U V E$ is an important research tool for research on dwarf novae because it is the first experiment with sufficient sensitivity and spectral resolution to be able to characterize the spectrum of the boundary layer region. A fundamental question, which one hopes to address with $E U V E$, is whether the boundary layer actually behaves in the manner that the conventional theory suggests. Is the boundary layer luminosity equal to that of the disk and what is the size of the emitting region? Three dwarf novae have been observed with the spectrometers aboard EUVE-U Geminorum, SS Cygni, and VW Hydri. Analysis of the observations of VW Hyi is not yet complete. Here, I will attempt to describe what has been learned thus far from the observations of U Gem and SS Cyg.

\section{U Geminorum}

U Geminorum, which lies a a distance of about $90 \mathrm{pc}$ (Marsh et al. 1990), is the prototypical dwarf nova, undergoing quasiperiodic outbursts $\left(\Delta \mathrm{m}_{\mathrm{v}} \sim 5\right)$ lasting typically 7-14 days about once every 118 days (Ritter 1990). In the far UV, the quiescent spectrum is dominated by the WD in the system (Panek \& Holm 1984). The average temperature of the WD surface drops from $\sim 38,000 \mathrm{~K}$ shortly after outburst to $\sim 30,000 \mathrm{~K}$ far from outburst (Long et al. $1993 ; 1994$ ). The $\sim 1 \mathrm{M}_{\odot}$ WD is rotating very slowly, if at all ( $\mathrm{v} \sin (\mathrm{i}) \leq 100 \mathrm{~km} \mathrm{~s}^{-1}$; Sion et al. 1994). In outburst, the far UV flux rises by a factor of 100 and is dominated by the optically-thick accretion disk (Panek \& Holm 1984). 


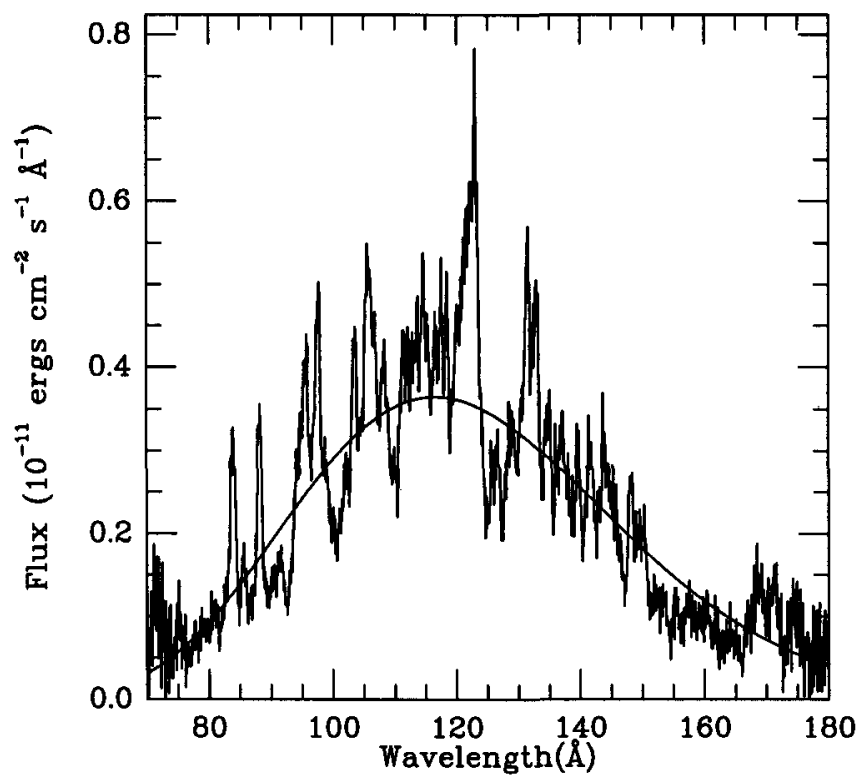

FIGURE 2. The fluxed spectrum of $U$ Gem as observed with the SW spectrometer during the second observing interval. The best fit blackbody model assuming $N_{H}=3 \times 10^{19} \mathrm{~cm}^{-1}$ is shown as the solid curve. The best fit temperature is $125,000 \mathrm{~K}$.

In outburst, $\mathrm{U}$ Gem is a bright soft $\mathrm{X}$-ray source, as evidenced by observations made with the soft X-ray proportional counters on HEAO-A by Córdova et al. (1984) and with the Einstein IPC by Córdova \& Mason (1984). Though the actual spectral shape was very uncertain given the very limited spectral resolution of the proportional counters being used, model fits to spectra were consistent with that expected from a blackbody having a temperature $\mathrm{kT}=10-\mathbf{3 0} \mathrm{eV}$. The luminosity of $\mathrm{U}$ Gem was not accurately determined by these observations; Córdova et al. (1984) quote values between $2 \times 10^{33} \mathrm{ergs} \mathrm{s}^{-1}$ and $1 \times 10^{35} \mathrm{ergs} \mathrm{s}^{-1}$. If the highest luminosity is correct and if the mass accretion rate was $8 \times 10^{-9} \mathrm{M}_{\odot} \mathrm{yr}^{-1}$ as was suggested by Panek \& Holm, then the luminosity of the disk is $8 \times 10^{34}(\mathrm{D} / 90 \mathrm{pc})^{2} \mathrm{ergs} \mathrm{s}^{-1}$ and it is quite possible that in U Gem the boundary layer and the accretion disk luminosities are similar. As predicted by the simple model, the $\mathrm{X}$-ray spectrum of $\mathrm{U}$ Gem hardens in quiescence.

Following reports from the AAVSO that an outburst of $U$ Gem had begun on the evening of 24 December 1993 and consultation with the $E U V E$ project scientist, three sets of observations of $\mathrm{U}$ Gem were carried out with the spectrometers on EUVE, beginning on 28 December and extending through 8 January 1994. The outburst was a typical "wide" outburst of the U Gem system, rising from quiescence to optical maximum in 2 days, remaining at maximum for $\sim 14$ days, and then declining to quiescence in $\sim 5$ days. The observations cover the peak and decline of the optical outburst. At peak, U Gem 
was one of the brightest sources in the EUV sky. As shown in Figure 1, long term (1 day) averages of the count rate in the SW spectrometer show a steady decline through the plateau phase of the optical outburst. During the first observing interval, the count rate in the $\mathrm{SW}$ spectrometer was about $5 \mathrm{cts} \mathrm{s}^{-1}$. A preliminary analysis of the data was presented by Long et al. (1995). The basic results are as follows:

The time-averaged spectra are quite complex, showing numerous emission and/or absorption lines, quite unlike that of most other types of objects-WDs, polars, or late-type stars-observed with high signal to noise with EUVE. The spectra do not resemble those of a thin plasma or a simple WD atmosphere. Assuming the emission peaks are emission lines then a substantial number of them can be identified with lines which would be present in a thin plasma ionization temperature of about $500,000 \mathrm{~K}$. Detailed model calculations will be required to properly interpret the spectra.

Mauche (1991) has measured the column density $\mathrm{N}_{\mathrm{H}}$ of cool interstellar material along the line of sight to U Gem to be $3 \times 10^{19} \mathrm{~cm}^{-2}$. Unit optical depth for this column density occurs at $95 \AA$, which accounts for the decrease in flux at long wavelengths. In order to make a rough estimate of the luminosity and size of the emitting region, the data were fitted to a blackbody spectrum assuming the absorption is given by the interstellar value. During the first observing interval, the apparent temperature U Gem was $\sim 134,000 \mathrm{~K}$ and the luminosity was $\sim 6 \times 10^{34} \mathrm{ergs} \mathrm{s}^{-1}$. Given this luminosity and temperature, the minimum size of the emitting region is $\sim 4.5 \times 10^{8} \mathrm{~cm}$, similar to that of a WD. If the EUV emission arises primarily from the boundary layer, then the boundary layer luminosity in U Gem is comparable to the disk luminosity, consistent with the standard theory of dwarf nova outbursts. The temperature declines as the outburst proceeds; fits to the spectra obtained in the second and third intervals yield $\sim 125,000$ and $\sim 100,000 \mathrm{~K}$ respectively. The characteristic size of the emitting region declines, but only slightly, to $\sim 3.0 \times 10^{8} \mathrm{~cm}$.

The count rates observed in both the SW and MW spectrometers are modulated on the orbital period, with broad a absorption dip near phase 0.7 , and additional structure near phase 0 (given an ephemeris in which secondary conjunction occurs at phase 0.0 ). These absorption dips had been observed with EXOSAT during a very anomalous 45 day long outburst (Mason et al. 1988). The new observation shows that the absorption dips are a common phenomenon in U Gem.

As shown in Figure 3, the lines in the spectrum appear to be less eclipsed than the continuum. This supports the hypothesis that the emission peaks are really lines and argues that the lines arise from an extended region while the continuum arises from the region of the WD.

The dips are surprising because of their phase and because the orbital inclination of $U$ Gem is relatively low $\left(\sim 65^{\circ}\right)$. The primary dip is not directly associated with the hot spot where the mass stream from the primary encounters the accretion disk which occurs near phase 0.9 . Mason et al. (1988), on the basis of one very rapid, nearly complete eclipse, suggested that the absorbing material must lie near the Roche lobe radius of the WD, considerably outside the corotation radius for the incoming mass stream, and a distance of $\sim 3 \times 10^{10} \mathrm{~cm}$ above the disk plane. Hirose, Osaki \& Mineshige's (1991) 3dimensional disk simulations do show that the outer regions of the disk have scale heights $\mathrm{H} \sim 0.1 \mathrm{r}$, an order of magnitude greater than estimates based on on hydrostatic balance. These models suggest the maximum scale height of the disk is $\sim 0.15 \mathrm{r}$ at phase 0.8 close to the phase at which the absorption dip is centered. In U Gem, however, the absorbing material has to lie approximately $0.42 \mathrm{r}$ from the disk mid-plane. The amount of material required to occult the source is quite small $\left(\mathrm{N}_{\mathrm{H}}<3 \times 10^{20} \mathrm{~cm}^{-2}\right)$, very much less than the surface density of the disk $\left(\mathrm{N}_{\mathrm{H}}>10^{24} \mathrm{~cm}^{-2}\right)$. 

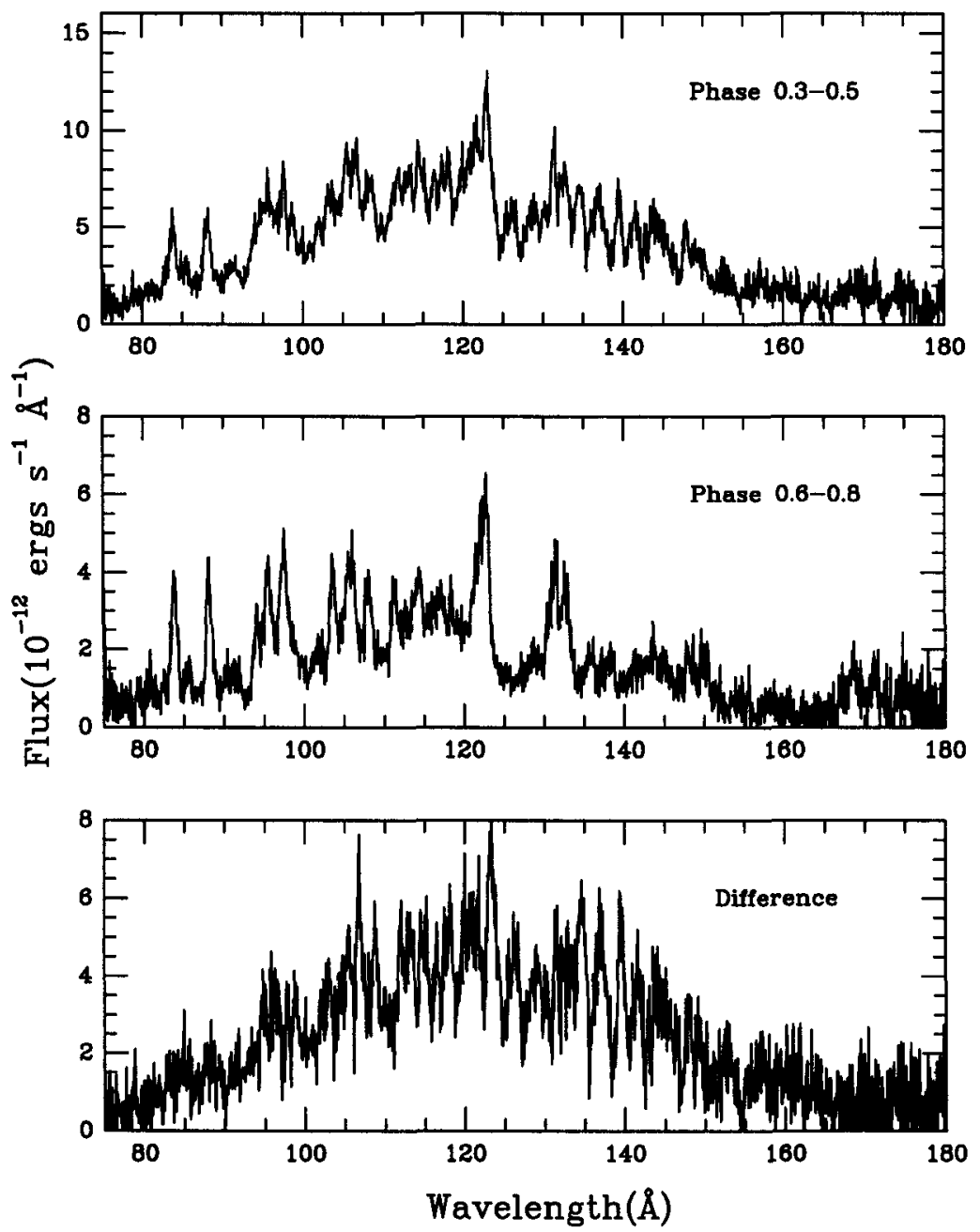

FIGURE 3. Fluxed SW spectra of U Gem from intervals 1 and 2 as a function of orbital phase. The EUVE emission is partially eclipsed near phase 0.7. The upper, middle and lower panels show the uneclipsed spectrum, the eclipsed spectrum, and the difference between the uneclipsed and eclipsed spectrum. The eclipse is mainly an eclipse of the continuum source.

\section{SS Cygni}

Like U Gem, SS Cyg is one of the brightest and best studied of dwarf novae. The system, as summarized by Ritter (1990), consists of $\mathrm{a} \sim 1.2 \mathrm{M}_{\odot} \mathrm{WD}$ and $\mathrm{a} \sim 0.7 \mathrm{M}_{\odot}$ K5 V star orbiting one another every 6.60 hour seen at an orbital inclination of $\sim 37^{\circ}$. During outbursts, which occur on average once every 49 days (Cannizzo \& Mattei 1992), the visual magnitude rises from 12 to values between 8 and 9. Although Holm \& Polidan 


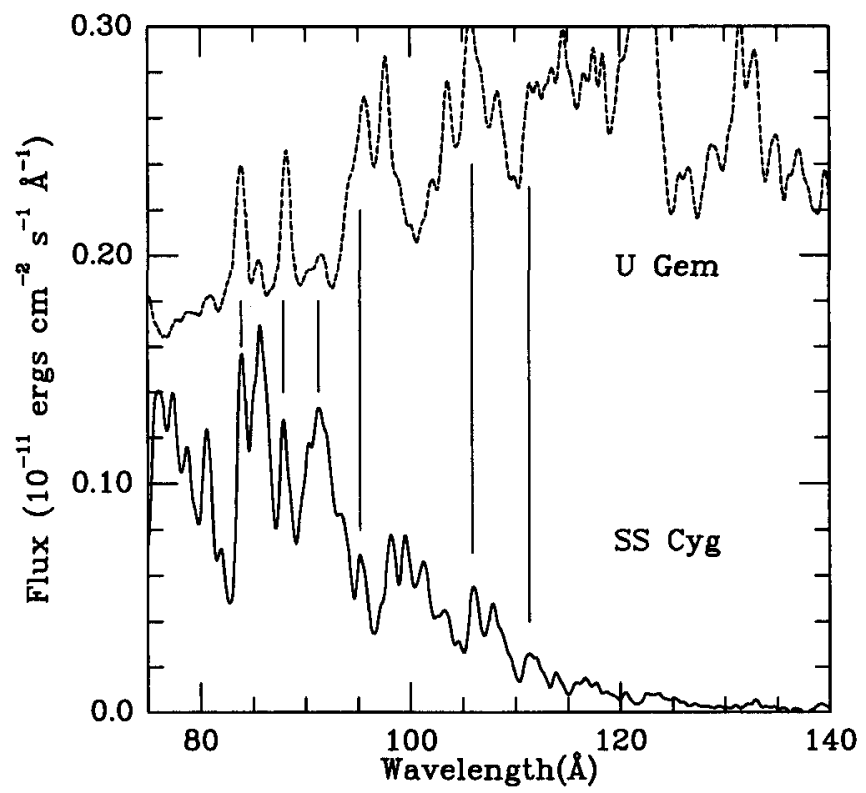

Figure 4. A comparison between the fluxed SW spectrum of SS Cyg and U Gem. The spectra have been convolved with a gaussian with a FWHM of $0.5 \AA$ to facilitate comparison of the two spectra.

(1988) have interpreted IUE spectra of SS Cygni in quiescence in terms of a WD with a temperature of $34,000 \pm 5000$, FUV (850-1850 $\AA$ ) spectra obtained with the Hopkins Ultraviolet Telescope show none of the absorption lines seen in spectra of $U$ Gem in quiescence and indicate that disk dominates the FUV emission in quiescence.

SS Cyg was the first dwarf nova to be detected at $\mathrm{X}$-ray wavelengths (Rappaport et al. 1974). In quiescence, $\mathrm{SS}$ Cyg is a bright soft $\mathrm{X}$-ray source; hard X-ray emission is generally suppressed, in basic agreement with the standard model (Jones \& Watson 1992), though not necessarily absent (Nousek et al. 1994).

SS Cyg has been observed twice with EUVE, first in 1993 August and then in 1994 June/July, Mauche and his collaborators; only the first observation has been reported in detail (Mauche, Raymond \& Mattei 1995), although a preliminary analysis of EUVE photometry of both outbursts is described in the volume (Mauche 1995). The discussion here is confined to the first outburst.

Though most outbursts in SS Cyg are classified as narrow or wide, depending primarily on the length of the outburst, the 1993 August outburst of SS Cyg was a a rare "symmetric" or "anomalous" outburst, in which the rise to outburst was exceptionally long $\sim 5$ days, comparable to the decay time. The observations began about 1 day after the beginning of the optical outburst and lasted $\sim 6.5$ days, covering the rise and the beginning of the plateau phase of the optical outburst. During the observation, the count 
rate in the SW spectrometer rose by about 2 orders of magnitude to a maximum rate of about $0.75 \mathrm{cts} \mathrm{s}^{-1}$. Surprisingly, the ratio of the count rate in the SW spectrometer in the wavelength range $90-130 \AA$ to the count rate in the range $72-90 \AA$ remained constant at a value of $1.39 \pm 0.05$ throughout the rise and during the peak portion of the outburst, suggesting a large change in the size of, but not the physical conditions in, the emitting region.

A comparison between the average spectrum obtained of SS Cyg and of U Gem (during the second observing interval) is shown in figure 4. Like U Gem, the spectrum of SS Cygni is complex and it is difficult to separate the continuum and the lines. There are however a number of "emission features" which are seen in both spectra. The "color" temperature of SS Cyg appears to be considerably higher than in U Gem., There is essentially no radiation detected from SS Cyg at wavelengths longer than $130 \AA$. It is not entirely clear whether this difference is due to absorption in the ISM or the vicinity of the source or due to the underlying source spectrum. Many of the features in the spectrum of SS Cyg are also seen in $U$ Gem.

Mauche et al. (1995) have also parameterized the spectrum in terms of a blackbody with cold intervening absorption. Estimates of $\mathrm{N}_{\mathbf{H}}$ from measurements of interstellar absorption lines $3.5 \times 10^{19} \mathrm{~cm}^{-2}$ are not not greatly different from those obtained for $\mathrm{U}$ Gem (Mauche, Raymond \& Córdova 1988), and yield a best 'fit' temperature of $37 \pm 2 \mathrm{eV}$ $(\sim 430,000 \mathrm{~K}), 3-4$ times that of $\mathrm{U}$ Gem in outburst. If one uses a blackbody parameterization of the system, and assumes a temperature of $20 \mathrm{eV}$, then $\mathrm{N}_{\mathrm{H}} \sim 7.0 \times 10^{19} \mathrm{~cm}^{-2}$, $\mathrm{L} \sim 2 \times 10^{33} \mathrm{ergs} \mathrm{s}^{-1}$ and the size of the emitting region is $3 \times 10^{-3}$ that of the WD surface. If $\mathrm{T}=30 \mathrm{eV}$, then $\mathrm{N}_{\mathrm{H}} \sim 4.4 \times 10^{19} \mathrm{~cm}^{-2}, \mathrm{~L} \sim 5 \times 10^{32} \mathrm{ergs} \mathrm{s}^{-1}$ and the size of the emitting region is $1 \times 10^{-4}$ that of the WD surface. The accretion disk luminosity in SS Cyg is $3 \times 10^{34} \mathrm{ergs} \mathrm{s}^{-1}$. If $E U V E$ is actually measuring the bulk of the boundary layer luminosity, which is not clear, then the ratio $\mathrm{L}_{\mathrm{bl}} / \mathrm{L}_{\text {disk }}$ is $\leq 0.07$ in SS Cyg, while in $\mathrm{U}$ Gem it appears to be closer to 1.

\section{Summary}

EUVE has been used obtain high S/N spectra of U Gem and SS Cyg (and VW Hyi). There are very significant differences in the spectra. It seems quite possible the boundary layer has been unmasked in both systems. Of the two dwarf novae which have been studied in detail, U Gem seems very consistent with the standard theory, while SS Cyg appears to provide a challenge.

None of the EUVE observations would have been possible were it not for the dedication of the observers of the AAVSO and of the mission support teams at Berkeley and at GSFC. I have benefitted greatly from discussions with Chris Mauche and John Raymond. My work on the EUV properties of dwarf novae has been supported generously by NASA's EUVE guest observer program (NAG5-2572).

\section{REFERENCES}

Cannizzo, J. K., \& Mattei, J. A. 1992, On the Long-Term Behavior of SS Cygni, ApJ, 401, 642

Cannizzo, J. K., \& WheEler, J. C. 1984, The Vertical Structure and Stability of AlphaModel Accretion Disks, ApJS, 55, 367

Cóndova, F. A., Chester, T. J., Mason, K. O., Kahn, S. M., \& Garmme, G. P. 1984, Observations of Quasi-Coherent Soft X-ray Oscillations in U Geminorum and SS Cygni, ApJ, 278, 739 
Córdova, F. A., \& Mason, K. O. 1984, X-ray Observations of a Large Sample of Cataclysmic Variable Stars Using the Einstein Observatory, MNRAS, 206, 879

Hirose, M., Osaki, Y., \& MiNeshige, S. 1991, Three Dimensional Structure of Accretion Disks in Close Binary Systems, Pub. Astr. Soc. of Japan, 43, 809

Holm, A. V., \& Polman, R. S. 1988, SS Cygni in Quiescence, in A Decade of UV Astronomy with the IUE Satellite, ESA SP-281, 179

Jones, M. H. \& WATSON, M. G. 1992, The EXOSAT, Observations of SS Cygni, MNRAS, 257,633

Long, K. S., Blair, W. P., Bowers, C. W., Davmsen, A. F., Kriss, G. A., Sion, E. M., \& HUBENY, I. 1993, Observations of the White Dwarf in the U Geminorum System with the Hopkins Ultraviolet Telescope, ApJ, 405, 327

Long, K. S., MaUche, C. W., SzKody, P., \& Mattei, J. A. 1995, EUVE, Observations of U Gem, in Proceedings of the Padova-Abano Conference on Cataclysmic Variables, in press

Long, K. S., Sion, E. M., Huang, M., \& Szkody, P. 1994, Cooling of the White Dwarf in U Geminorum Between Outbursts, ApJL, 424, L49

Marsh, T. R., Horne, K., Schlegel, E. M., Honeycutt, R. K., \& Kaitchuck, R. H. 1990, ApJ, 364, 637

Mason, K. O., Córdova, F. A., Watson, M. G., \& King, A. R. 1988, The Discovery of Orbital Dips in the Soft X-ray Emission of U Gem During an Outburst, MNRAS, 232, 779

MAUCHE, C. W. 1991, private communication

MAUCHE, C. W. 1995, EUVE, Photometry of SS Cygni: Dwarf Nova Outbursts and Oscillations, these proceedings

Mauche, C. W., Raymond, J. C., \& Córdova, F. A. 1988, Interstellar Absorption Lines in High Resolution IUE, Spectra of Cataclysmic Variables, ApJ, 335, 829

Mauche, C. W., Raymond, J. C., \& Mattei, J. A. 1995, EUVE, Observations of the Anomalous 1993, Outburst of SS Cygni, ApJ, 446, in press

Meyer, F., \& MEYer-Hofmeister, E. 1992, Vertical Structure of Accretion Disks, A\&A, 106,34

Nousek, J. A., Baluta, C. J., Corbet, R. H. D., Mukai, K., Osbourne, J. P. 1994, ASCA, Observations of SS Cygni During an Anomalous Outburst, ApJL, 436, L19

OsaKI, Y. 1974, An Accretion Model for the Outbursts of U Geminorum Stars, Pub. Astr. Soc. of Japan, 26, 429

PANEK, R. J., \& Holm, A. V. 1984, Ultraviolet Spectroscopy of the Dwarf Nova U Geminorum, ApJ, 277, 700

Pringle, J. E. 1977, Soft X-ray Emission from Dwarf Novae, MNRAS, 178, 195

Rappaport, S., Cash, W., Doxsey, R., McClintock, J., \& Moone, G. 1974, Possible Detection of Very Soft X-rays from SS Cygni, ApJL, 187, L5

RitTer, H. 1990, Catalogue of Cataclysmic Binaries, Low-Mass X-ray Binaries \& Related Objects, A\&AS, 85, 1179

Sion, E. M., Long, K. S., Szkody, P., \& Huang, M. 1994, Hubble Space Telescope, Goddard High Resolution Spectrometer Observations of U Geminorum in Quiescence: Evidence for a Slowly Rotating White Dwarf, ApJL, 430, L53 